\title{
Pancreatoblastoma Mimicking Liver Abscess: A Case Report.
}

\author{
Z Syed $^{1}$, S Chakravarthy ${ }^{1}$, MSingh $^{1}$, R Narayan $^{2}$ \\ ${ }^{1}$ Department of Radiology, ${ }^{2}$ Pathology, Tata Main Hospital, Jamshedpur, Jarkhand.India
}

\begin{abstract}
Pancreatoblastoma is an extremely rare pancreatic tumour most commonly encountered in infants and young children. This is a case of childhood pancreatoblastoma being reported to emphasize the dilemma of clinical and radiological features in diagnosing this rare tumour. A 3 -year-old boy complained of distension of the abdomen with mild jaundice. Initially, physical and radiological investigations were in favour of liver abscess. Histopathological examination of the resected specimen was interpreted as liver mass. Serological investigations and immunohistochemistry for malignancy were helpful in suspecting Pancreatoblastoma. Pancreatoblastoma can mimic a liver mass (caudate lobe) on imaging as is shown in this case report.
\end{abstract}

Keywords: Alpha-fetoprotein, Hepatoblastoma, Pancreatoblastoma, CK= Cytokeratin, EMA= Epithelial membrane antigen.

\section{Key message}

Pancreatoblastoma, though rare, should be kept in mind whenever dealing with unexplained epigastric mass in a young child. Histopathology is mandatory for confirming the diagnosis. Immunohistochemistry should be done in diagnostic dilemma.

\section{Introduction}

Pancreatoblastoma was first described by Frantz in 1959. Subsequently, Frable et al in 1971 described the histological and ultrastructural findings and called it "infantile carcinoma of pancreas". This tumour is considered to be embryonic in origin, based on the histological appearance. It accounts for $0.5 \%$ of exocrine tumors of the pancreas and the highest incidence is found in the first

Correspondence to: Dr Zubair Syed, Department of Radiology, Tata Main Hospital, Jamshedpur, Jharkhand, India.

E-mail: docshahzubair@gmail.com decade of life with a predilection for males and Asians. ${ }^{1,2}$ In approximately two-third of cases increased alpha-fetoprotein levels is found, which also happens in hepatoblastoma and other blastomal carcinomas. Congenital pancreatoblastomas are associated with Beckwith-Weidman syndrome. ${ }^{3}$ Most pancreatoblastomas exhibit typical genetic alterations similar to those occurring in hepatoblastoma and acinar cell carcinoma.

\section{Case Details}

3yr old male child admitted with pain abdomen for 1 day; fever off $\&$ on 1 week and yellowish discolouration of body for 2 weeks. On examination abdomen was tender $\&$ distended with mild hepatomegaly. A firm mass was palpable in epigastric region not separable from liver margin. Spleen was not palpable. Bowel sounds were audible. Chest, CVS \& CNS were unremarkable. 


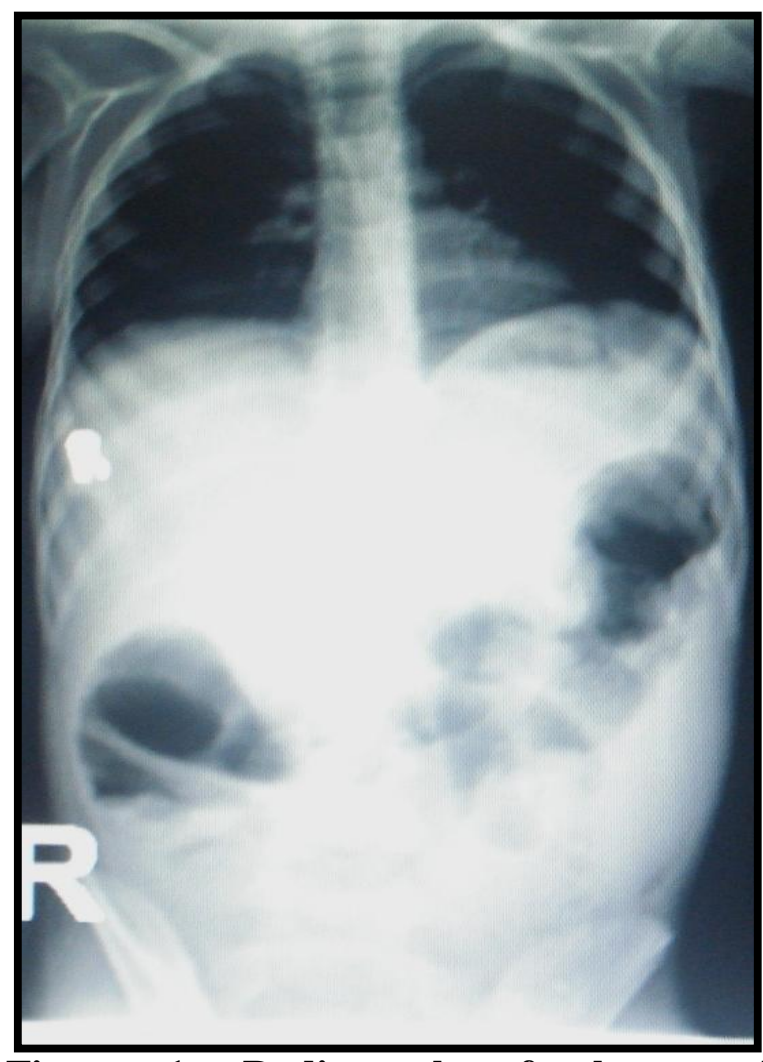

Figure 1: Radiograph of chest and abdomen of a $3 \mathrm{yr}$ old child, centered at diaphragm. Note the displaced bowel loops from epigastric region.

Haematology: $\mathrm{CBC}$ and $\mathrm{PBF}$ revealed normal picture. No parasite seen on thin and thick

smears.

Biochemical: Sr. Bilirubin- $1.7 \mathrm{mg} / \mathrm{dl}$, ALT59.0 U/L, ALP- 839.2U/L.

$X$ ray abdomen PA view (Fig 1):- Grossly normal with displaced bowel loops from epigastric region.

USG Abdomen: Liver was enlarged in size with multiple hypoechoic lesions $(3 \mathrm{~cm}$ to 6 $\mathrm{cm})$ seen in both lobes, largest one located in the caudate lobe. Epigastric and periportal lymph nodes were enlarged with mild ascites. Rest of abdominal organs were unremarkable.

Impression: Multiple liver abscesses with ascites and lymphadenopathy.

CECT abdomen: CECT abdomen showed multiple, irregular, hypodense, grossly non- enhancing areas in liver with mildly enhancing rim, largest one located in the caudate lobe. Left branch of portal vein showed filling defect. Spleen, pancreas and kidneys looked normal. Enlarged nodes were seen at the porta and epigastric region. Moderate free fluid was seen in peritoneal cavity.

Impression: Multiple hepatic abscesses with portal vein thrombus (left branch), enlarged portal, perigastric lymph nodes and ascites. Patient was referred to the paediatric surgeon, who, on laparotomy, drained $700 \mathrm{ml}$ of blood tinged fluid from peritoneal cavity. No histopathological examination. visceral or gut injury was found. Pieces of liver tissue were seen in the fluid. A ruptured necrotic liver pathology was suspected and tissue specimens were sent for histopathological examination.

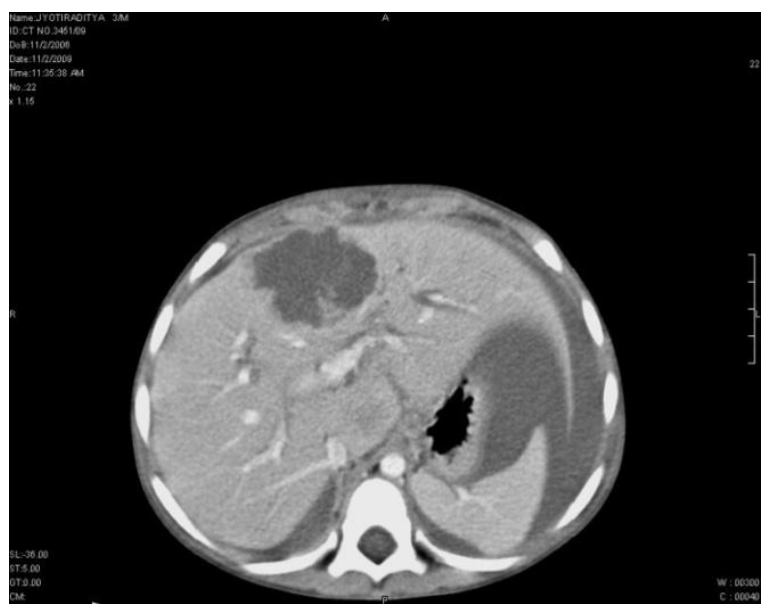

Figure 2: CECT abdomen(venous phase, $5 \mathrm{~mm}$ slice) of a $3 \mathrm{yr}$ old child showing irregular hypodense lesion with mildly enhancing rim \& a small area posteriorly in segment 8 of right lobe of liver. Note the focal narrowing of left branch of portal vein and ascites.

Microscopic pathological examination showed a cellular tumour with sheets of pleomorphic round to oval cells, vesicular nuclei and prominent nucleoli separated by fibroconnective stromal tissue. Focal acinar arrangement of tumour cells with atypical 
mitosis and areas of necrosis were seen. Impression of Hepatoblastoma was made.

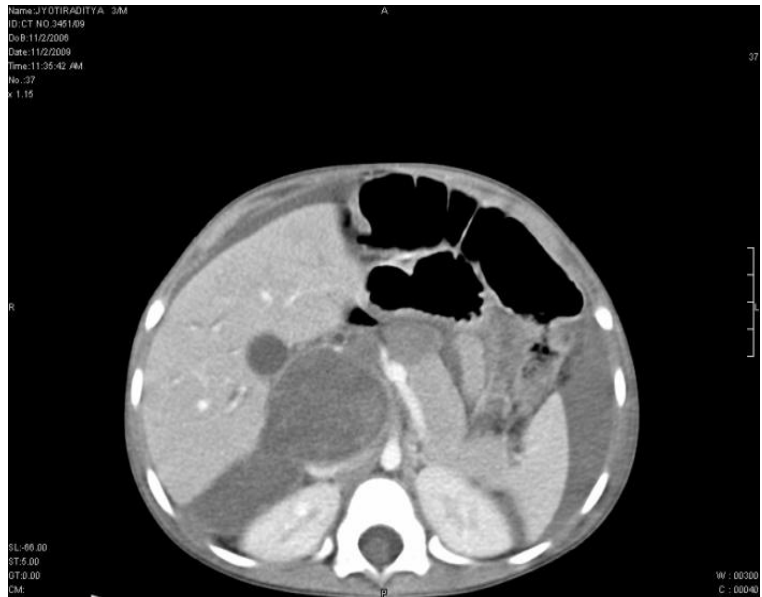

Figure 3: CECT abdomen (venous phase, $5 \mathrm{~mm}$ slice using spiral CT) at the level of portal confluence. Note the large well defined hypodense area compressing and displacing the liver and pancreas and adjacent vessels, portal vein anteriorly and IVC posteriorly. Lesion shows rim enhancement with faintly enhancing some solid component. It is pancreatoblastoma arising from head of pancreas. Also note some periportal nodes.

Multiple extensively necrotic liver lesions with perigastric lymphadenopathy and no significant rise in serum alpha-fetoprotein are not features of a typical Hepatoblastoma, so Immuno-histochemistry of tissue samples was done which showed tumour cells positive for cytokeratin \& epithelial membrane antigen and negative for alpha-feto protein. Thereafter pancreatoblastoma was suspected.

Abdominal CT scan (Fig. 2 \& 3) was again reviewed for the origin of epigastric mass. The mass was seen related to the caudate lobe of liver and the pancreatic head but as the pancreatic head was not seen separately (Vanishing sign) so it was actually arising from the pancreas and compressing the liver. Keeping all features in view a retrospective diagnosis of Pancreatoblastoma with metastasis to liver was made.
Patient is now on chemotherapy for pancreatoblastoma.

\section{Discussion}

Pancreatoblastoma is a slow growing, well circumscribed, usually very large tumour at the time of diagnosis ${ }^{1,3}$. Approximately half of the tumors are found in the pancreatic head4. Pancreatoblastomas share a similar radiological appearance in both adult and paediatric patients1. Most of the tumors are grossly inhomogeneous due to coexisting solid and cystic areas. Septations and calcification can also be present ${ }^{1,4}$.

In paediatric patients, very large pancreatoblastomas cannot be differentiated radiologically from hepatoblastoma or other infantile tumors. ${ }^{4}$ MRI delineates intratumoral haemorrhage and necrosis much better. On the other hand, CT better demonstrates calcium deposits, which usually appear as rim calcifications or foci of punctuate calcifications. ${ }^{4}$ Hepatic, lymph nodal and peritoneal metastases are evident in approximately $30 \%$ of cases on clinical presentation. Pulmonary and bone metastases have also been reported. ${ }^{3}$

A pancreatic pseudocyst can have inhomogeneous contents and peripheral calcification, but a history of pancreatitis and the absence of solid areas often lead to the correct diagnosis. ${ }^{4}$

Mucinous cystic neoplasms are predominantly cystic. Calcifications and fluid level from haemorrhage can exist but the enhancement of the capsule is typically delayed. $^{5}$

Surgery remains the treatment of choice for both the primary tumor and the metastases. ${ }^{3}$ An empirical approach with chemotherapy can also play a role in the planning of treatment whereas radiation therapy is indicated in the case of incomplete surgical resection.$^{2}$ 


\section{Conclusion}

It was difficult to call this mass lesion as pancreatoblastoma in first place because of rare occurrence, extensive necrosis and multiplicity, resembling multiple liver abscesses. Being a very large mass, the origin of the mass was difficult to establish on imaging. The features pointing towards pancreablastoma would have been a large mass related to head of pancreas with positive vanishing sign, enhancing solid component in the mass, similar appearing masses in the liver, involvement of portal vein and enlarged local nodes. Pancreatoblastoma is a rare tumor but should be considered in a child with unexplained epigastric mass.

\section{References}

1. Chung EM, Travis MD, Conran RM. Pancreatic tumors in children: radiologic-pathologic correlation. Radiographics 2006; 26:1211-38.
2. Saif MW. Pancreatoblastoma. JOP. J Pancreas (Online) 2007; 8:55-63.

3. Klimstra DS. Nonductal neoplasms of the pancreas. Mod Pathol 2007; 20:94-S112.

4. Montemarano $\mathrm{H}$, Lonergan GJ, Bulas DI, Selby DM. Pancreatoblastoma: imaging findings in 10 patients and review of the literature. Radiology 2000; 214:476-82.

5. Cantisani V, Mortele KJ, Levy A, Glickman JN, Ricci P, Passariello R, et al. MR imaging features of solid pseudopapillary tumor of the pancreas in adult and pediatric patients. AJR Am J Roentgenol 2003; 181:395-401. 